\title{
An Unusual Neuroendocrine Tumor
}

\author{
Armeen Lakhani ${ }^{*}$, Naila Zahid ${ }^{1}$ and Mirza Arshad Beg² \\ ${ }^{1}$ Department of Oncology, Liaquat National Hospital and Medical College, Karachi, Pakistan \\ ${ }^{2}$ Department of General Surgery, Liaquat National Hospital and Medical College, Karachi, Pakistan
}

\begin{abstract}
This is a case of a 62-year-old male, presented with UGI symptoms for a short period, upon biopsy it turned out to be a neuroendocrine tumor with hepatic metastasis and locoregional nodes.
\end{abstract}

Keywords: Neuroendocrine, histopathology, tumor.

\section{INTRODUCTION}

A unique group of malignant growths NEUROENDOCRINE TUMORS, best known for their ability to secrete bioactive peptides, which may cause symptoms such as flushing and diarrhea [1]. NETs classified as clinically symptomatic (functioning) or silent (nonfunctioning); both types frequently synthesize more than one peptide, although often these are not associated with specific syndromes [2]. As they being uncommon and may be nonspecific in their prentation, delays in diagnosis are frequent.

\section{CASE PRESENTATION}

A 62-year-old male known comorbid, presented in Oncology OPD with complaints of abdominal pain, nausea, vomiting and weight loss since 2 months. On examination, he had mild to moderate hepatomegaly almost 4 finger breadths from the costal margin with no tenderness, and the rest of the abdomen was soft with no distention or so. The rest of the systemic examination was normal. Investigations have been done to make the diagnosis.

\section{RADIOLOGICAL FINDINGS-CT ABDOMEN WITH CONTRAST}

The liver was enlarged with multiple hypodense areas (see Fig. 1). One of them was at anterior superior sub-segment viii with a lesion of $5.1 \times 2.1 \mathrm{~cm}$ with no intrahepatic biliary duct dilatation. There was abdominal lymphadenopathy with enlarged lymph nodes in the peripancreatic and mesenteric region-largest: $3.2 \mathrm{~cm}$. There was minimal thickening of the pyloric end of the stomach.

\section{BONE SCAN}

Negative for any metastasis but arthritic changes in the left knee joint.

\footnotetext{
*Corresponding Author: Armeen Lakhani, Department of Oncology, Liaquat National Hospital and Medical College, Karachi, Pakistan, Email:armeenlakhani@yahoo.com

Received: October 02, 2020; Revised: February 03, 2021; Accepted: February 15, 2021
} DOI: https://doi.org/10.37184/lntbj.2708-7808.2.12

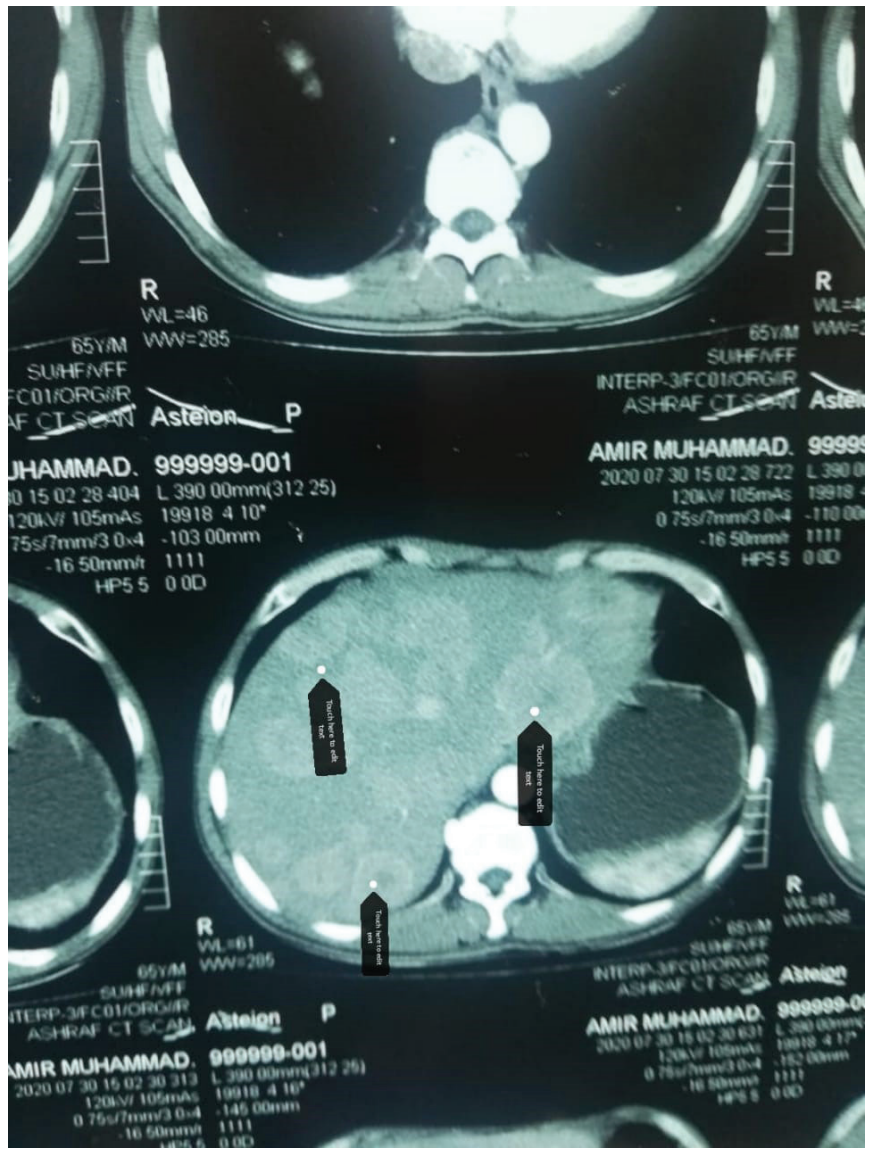

Fig. (1): Enlarged liver with marked pointers as hypodense lesions in liver.

\section{ENDOSCOPIC FINDINGS}

The patient had undergone EGD and Biopsy in which at the stomach there was a large irregular mass at the body of the stomach extending from lesser curvature to the pylorus (see Fig. 2). Multiple biopsies were taken.

\section{HISTOPATHOLOGY DETAILS}

The gastric growth biopsy showed individual cells with round nuclei with coarse chromatin and pale cytoplasm. The Immuno Histo Chemistry showing CK AE1/ AE 3 and SYNAPTOPHYSIN POSI-TIVE WITH Ki 67 of 10- 


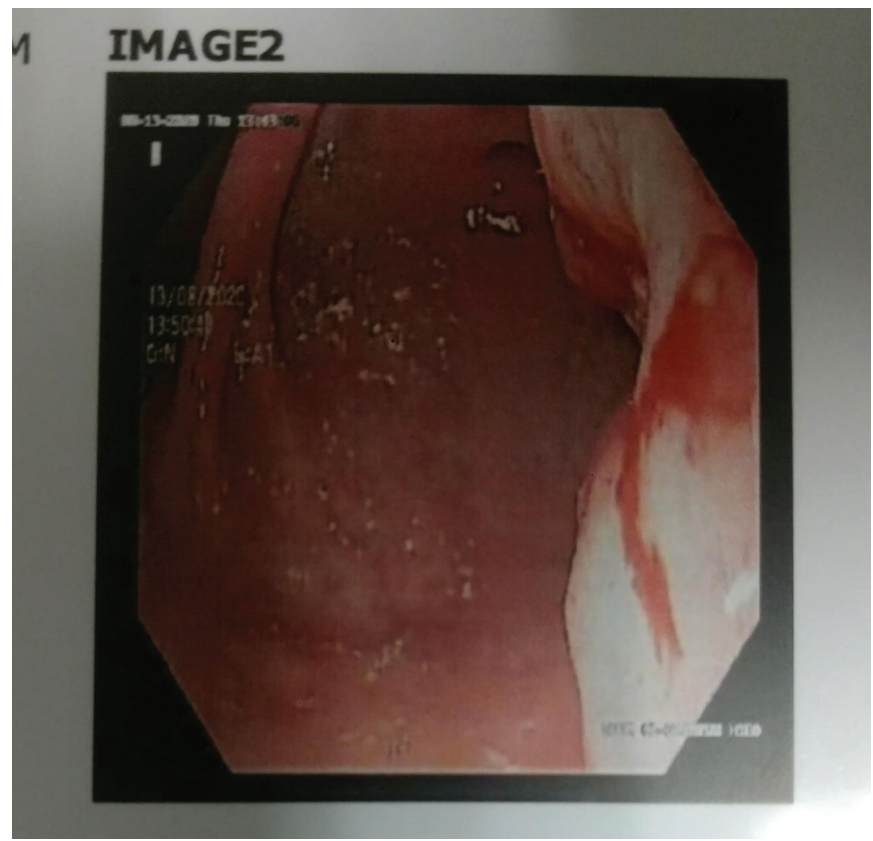

Fig. (2): Large irregular mass at the body of stomach, extending from lesser curvature to pylorus.

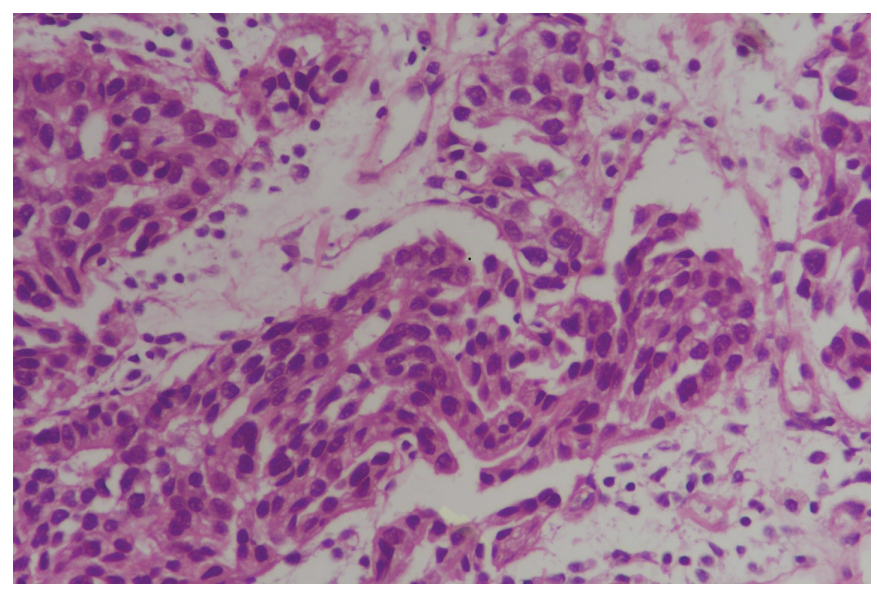

Fig. (3): Nests of tumor cells with rounded nuclei showing coarse chromatin and pale cytoplasm.

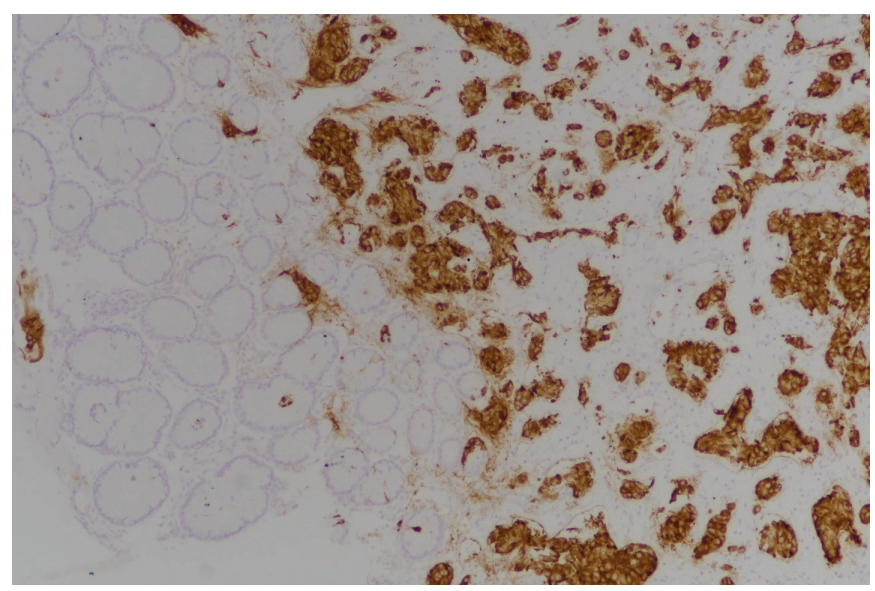

Fig. (4): The immunohistochemistry showing $C K A E 1 / A E 3$ and synaptophysin positive with Ki 67 of $10-15 \%$.
15\% (see Figs. 3 \& 4) Favoring neuroendocrine tumor - grade II.

\section{TUMOR BOARD DISCUSSION}

In the multidisciplinary meeting involving Medical Oncologists, General Surgeons, Histo-pathologists and Radiologists case was discussed and options of surgery versus systemic therapy were discussed. Surgeon Dr. Nadeem Khurshaidi raised the issue of the possibility of adeno carcinoma also as that will completely change the prognosis. The histopathologist Dr. Hanna Naqvi responded by saying that it's very unlikely because Synaptophysin is strongly positive. Dr. Nadeem Khurshaidi said in a patient with multiple liver metastases and lymphadenopathy the indication for resection of the primary tumor is only if the patient is symptomatic with an ob-struction or GI bleed and the patient doesn't have any of these. Dr. Misbah from radiology ad-vised to get DOTATATE SCAN done to further clarify disease activity and extent. Dr. Naila Zahid asked for the role of TACE but the consensus was because of the extent of liver metastases its benefit is questionable. Therefore Board decided to get a Dotatate scan and urinary 5 HIAA and then start Somatostatin analogues.

\section{SURGEON'S PERSPECTIVE}

Literature is scant regarding management protocols for Gastric Neuroendocrine tumors with liver metastasis. These are a set of rare tumors that secrete neuroendocrine hormones and have the potential of metastasis. Upon presentation, almost 40 to 70 percent of tumors have already metastasized to the liver. Liver Metastasis accompanies about $28.3-77.0 \%$ of pancreatic NET and $67-91 \%$ of small intestine NET at initial presentation [3], which is an important prognostic marker.

Surgical resection remains the mainstay of therapy in such tumors even with liver metastasis. In patients receiving complete surgical resection, the 5 -year survival rate may reach up to 80 percent in contrast to the nonsurgical approach which is only 30 percent [4].

Based on liver metastasis finding, the distribution is as follows in groups $[4,5]$ :

I. Type I: for liver metastases localized in one lobe or two adjacent segments, standard anatomical resection can achieve the en bloc resection of the lesion. Such cases of GEP-NET with liver metastasis account for about $20-25 \%$.

II. Type II: the metastatic lesions are mainly distributed in the right or left lobe of the liver; however, small satellites still exist in another lobe. This type accounts for about $10-15 \%$. Clinically this type is mainly treated with multidisciplinary strategies including surgical resection (the mainstream treatment) and ablation therapy. 
III. Type III: the metastases are diffusely distributed in the left and right lobes of the liver and cannot be treated with surgery. This type accounts for about $60-70 \%$.

Generally, the surgical treatment of GEP-NET with liver metastasis must meet the following conditions: (i) with resectable well-differentiated (G1/G2) liver metastases and the predicted peri-operative mortality rate of $<5 \%$; (ii) without right cardiac dysfunction; (iii) without unresectable lymph node metastasis or extraperitoneal metastases; and (iv) without diffuse or unresectable peritoneal metastasis [6].

In our current case, the patient already has gNEN (G2) with multiple liver metastases at first presentation. In this scenario, we may consider it to be a type III liver metastases. In such a case according to case reports in the literature, as no randomized trial is available a treatment strategy with somatostatin analogues and ablation therapy may be considered fol-lowed by surgery as palliative resection may still have a role in such cases. However, the prognosis in these cases is guarded despite the best efforts.

\section{ONCOLOGIST PERSPECTIVE}

Gastric NETs consist of a complex disease that includes different subtypes with distinct management and prognosis. Poor prognosis factors are lesion $\geq 2 \mathrm{~cm}$; deep submucosa invasion or beyond (at least $24 \%$ are metastatic); Ki-67 $\geq 3 \%$; vascular invasion; a low degree of structural differentiation; presence of atypia and/or necrosis. There are four types of gastric neuroendocrine tumors and management varies accordingly.

\section{Type I}

It comprises $70-80 \%$ of gastric NET and is associated with autoimmune chronic atrophic gastritis destroying the gastric parietal cell, and subsequent achlorhydria, which in turn results in hypergastrinemia leading to ECL cells hyperplasia. The typical endoscopic finding is multiple reddish polyps. The proliferation marker MKI67 is typically expressed in less than $2 \%$ of the tumor cells. Patients usually have hypergastrinemia and B12 deficiency [7]. It has a good prognosis and endoscopic resection is sufficient treatment [8]. Netazepide (YF476) is a gastrin receptor antagonist. YF476 has been reported to cause tumor regression in Type I gastric NET [9].

\section{Type II}

$7 \%$ of gastric NET are type II. It is associated with Zollinger Ellison syndrome resulting in hyper-gastrinemia and hyperchlorhydria. Endoscopy shows normal or hypertrophic gastric mucosa. The proliferation marker MKI67 is expressed in $<2 \%$ of the tumor cells. Usually, the tumor is near the head of the pancreas, between the second and third part of the duodenum or near the junction of the cystic duct with a common hepatic duct. It has a very little chance of metastases alt-hough slightly more aggressive than type1. Treatment consists of resection of gastrinoma and endoscopic resection of gastric polyp if any [10]. In patients with multiple tumors that can't be resected endoscopically, Somatostatin analogue octreotide can be used and results in a marked decrease in plasma gastrin levels with subsequent tumor regression [11].

\section{Type III}

This is the sporadic type and is $14 \%$ to $25 \%$ of gastric NET. It is associated with normal Gastrin level. Endoscopy shows a single lesion usually greater than $1 \mathrm{~cm}$ in normal gastric mucosa. More than $2 \%$ of tumor cells express the proliferation marker MKI67. This type has a high propensity to metastasize and is associated with worse 5-year survival of 75 to $80 \%$ compared with 90 to $95 \%$ for type I tumors. Rarely carcinoid syndrome can be the presenting symptom especially in patients with liver metastases.

\section{Type IV}

Type IV GNET is an uncommon tumor, and usually is single, large, poorly differentiated, and highly malignant; it is typically accompanied by vascular invasion and metastases, has high levels of MKI67 (> 30) and has an extremely poor prognosis with mean survivals of 6.5 to 14.9 months. The mainstay of treatment is aggressive surgery followed by chemotherapy [12]. The commonest site of metastases of neuroendocrine tumors is the liver. In a Swedish study, $82 \%$ of patients with metastatic Neuroendocrine tumors had liver metastasis and the commonest source was the small intestine. The risk of metastasis was highest if the primary was in the small intestine or pancreatic hepatobiliary tract, whereas it was lower with appendiceal and rectal NET [13].

Prognostic factors in patients with the metastatic disease include disease burden, the presence of distant extrahepatic metastases, race, and older age. Biochemical characterization of the tumor can help in further characterization of the tumor and future monitoring of response to treatment.

In general 24- hour urine or plasma HIAA should be checked whereas in patients with gastric NET serum gastrin level should be checked, and in pancreatic NETs VIP, serum Insulin and serum Glucagon should be checked. The "gold standard" for imaging any individual suspected of NET dis-ease is Gallium 68-Dotatoc (68Ga) PET-CT. It can show evidence of both primary and secondary disease with high specificity [14]. In patients with metastatic disease, it is important to find the primary to avoid side effects related to primary i.e. obstruction, bleeding etc.

Metastatic neuroendocrine tumor, the treatment of metastatic NET is multimodality. Since even metastatic NET usually has an indolent course the goal of treatment is usually control of symptoms and maintain quality of life rather than cure. It can be divided into systemic and locoregional which intern can be surgical or non- 
surgical. Systemic treatment can also be divided into two tiers: symptomatic control and control of tumor growth. Somatostatin analogues play an important role in controlling symptoms [15]. Octreotide and lanreotide have shown similar efficacy in terms of carcinoid syndrome palliation, with symptom control rates usually ranging between 45 and $75 \%$. In case of worsening of symptoms before the next due dose frequency of drug ad-ministration can be increased or an extra dose of short-acting octreotide may be effective. In patients with refractory carcinoid syndrome telotristat ethyl Serotonis synthesis inhibitor as-sessed in two doubleblind, placebo-controlled, phase III TELESTAR [16] and TELECAST trials at 250 or $500 \mathrm{mg}$ significantly reduced bowel movement frequency and 5-HIAA levels. Telotristat is generally well-tolerated, is associated with mild nausea and hepatic enzyme elevations.

Somatostatin analogues are very effective in slowing the disease progression also. In phase III PROMID trial monthly Octreotide LAR 30mg resulted in statistically and clinically significant improvement in median time to progression from 6 months on the placebo arm to 14.3 months on the octreotide arm (hazard ratio 0.34; $p=0.000072$ ) [17]. Similar results were found in another double-blind, placebo-controlled, phase III CLARINET trial of 204 patients with lanreotide depot at $120 \mathrm{mg} / 4$ weeks or placebo with $96 \%$ of patients having radiographically stable disease. Lanreotide was associated with a significant prolongation of progression-free survival (PFS) when com-pared with placebo (median not reached versus 18 months, after a median study drug exposure of 24 months; hazard ratio $0.47, p<0.001$ ) [18]. Although both the trials had only patients with Ki-67 index of less than $10 \%$, likely, both octreotide and lanreotide are also active in welldifferentiated, SSTR-positive tumors whose proliferative activity exceeds $10 \%$.

Radiolabeled somatostatin analogs are a form of radiolabeled systemic therapy. Phase III NET-TER-1 study of 177Lu-DOTATATE versus high-dose octreotide LAR (60 mg/month) in 229 patients with advanced, octreoscan-positive midgut NETs who progressed on standard-dose octreotide LAR showed that 177LuDOTATATE therapy resulted in a $79 \%$ reduction in risk of progression or death compared with high-dose octreotide $(p<0.0001$; hazard ratio $0.21 ; 95 \% \mathrm{Cl}, 0.13-0.33)$ at 14 months follow up. The median PFS was not reached in the lutetium arm versus 8.4 months in the control arm, and consistent benefit across major subgroups was shown by subgroup analysis. The overall response rate (ORR) observed in patients treated with PRRT was significantly higher than in patients who received octreotide (18 versus $3 \%$, respectively; $p<0.0004$ ) [19]. Although the short time effects including nausea, vomiting and myelosuppression are manageable its limitations are the long-term toxicities including renal failure and leukemia/myelodysplastic syn-dromes
(MDS). The long term incidence of acute leukemia/ MDS after PRRT is 2\%. M-Tor inhibitor Everolimus previously approved for Pancreatic NET was found to have a statistically significant benefit in PFS in NETs of lung and gastrointestinal origin in double-blind, phase III RADIANT-4 study from 3.9 months on the placebo arm to 11 months on the everolimus arm (hazard ratio $0.48 ; p$ $=0.00001$ ) leading to FDA for the treatment of advanced NETs, regardless of the primary site [20]. Side effects of the mTOR inhibitor include hyperglycemia, cytopenias, oral ulcers, rash, diarrhea, and atypical infections, including potentially serious pneumonitis.

Antiangiogenic agents have been found to have activity in pancreatic NETS. There is an ongoing trial with sunitinib in gastrointestinal NETs. IFN has shown both antisecretory and antiproliferative activities in GEPNETs. In one study addition of IFN to octreotide showed improvement in symptom control in $49 \%$ of patients with suboptimally controlled carcinoid syndrome [21]. In patients with poorly differentiated neuroendocrine carcinomas chemotherapy should be considered. Usually like small cell lung cancer there is a rapid response to platinum-based regimens, but remissions are usually short-lived. Thus far, no studies have compared platinumbased regimens versus temozolomide- or streptozocincontaining protocols in patients with poorly differentiated GEP-NETs. Combination of CDDP plus irinotecan (CPT11) has an overall response rate of $75 \%$ and progressionfree survival of $212 \mathrm{~d}$ [22]. Chemotherapy is found to be effective in, low-to-intermediate-grade pNETs who have bulky, rapidly progressive, and/or symptomatic disease but minimal activity in gastrointestinal NET.

There are ongoing trials with PD1 monoclonal antibodies. Pancreatic NETs have a worse prognosis than GINETs and respond differently to anticancer agents, with most agents demonstrating higher response rates among patients with pancreatic NETs than those with GINETs. Further-more, survival is better in small intestinal primary compared to colon primary.

Locoregional Treatment in patients with metastatic NETs, because of longer median survival as compared to other malignancies resection of the primary should be considered if the primary site is causing symptoms. Moreover in patients with a resectable primary and liver metastases but no extrahepatic disease, diffuse bilobar involvement, or compromised liver function surgical resection of the primary, as well as metastasectomy, should be performed. On the contrary, it's unclear if the resection of asymptomatic primary sites in patients with unresectable metastatic disease is of any benefit. Surgical resection of liver metastases not only provides symptomatic control, in some studies is found to be associated with a survival benefit (50 to $60 \%$ 10-year survival) but this is proportional to the extent of resection of the tumor burden [23]. Whereas for patients who have multifocal and bilateral disease needing major hepatectomy with a risk of major compromise in function 
of the remaining liver, ablation is most often used as an adjunct to surgical resection to allow local treatment of all disease which is associated with $50 \%$ response rate [24]. Surgery is contraindicated in metastatic poorly differentiated neuroendocrine carcinoma because of its poor prognosis.

\section{CONCLUSION}

In metastatic neuroendocrine tumors with isolated liver metastases resection of the primary tumor and liver metastases can provide symptom control and has been associated with favorable long-term survival, with 10year survival rates approaching 50 to 60 percent in some series. In patients with unresectable disease, treatment decision depends on symptoms, tumor characteristics and performance status of the patient. In patients with low-grade tumors Somatostatin analogues not only can control the symptoms but also improve PFS. Whereas in rapidly progressive poorly differentiated tumors earlier use of chemotherapy and radiolabeled somatostatin analogues is warranted.

\section{CONFLICT OF INTEREST}

The authors delcare no conflict of interest.

\section{ACKNOWLEDGEMENTS}

Declared None.

\section{REFERENCES}

1. Raphael MJ, Chan DL, Law C, Singh S. Principles of diagnosis and management of neuroendo-crine tumours. Can Med Assoc J 2017; 189: E398-E404.

2. Öberg KE. Management of neuroendocrine tumors: current and future therapies. Expert Rev Endocrinol Metab 2011; 6: 49-62.

3. Frilling $A$, Modlin $I M$, Kidd $M$, et al. Recommendations for management of patients with neur endocrine liver metastases. Lancet Oncol 2014; 15: e8-21.

4. Pavel M, Baudin E, Couvelard A, et al. ENETS Consensus Guidelines for the management of patients with liver and other distant metastases from neuroendocrine neoplasms of foregut, midgut, hindgut, and unknown primary. Neuroendocrinology 2012; 95: 157-76.

5. Panzuto $F$, Nasoni S, Falconi M, et al. Prognostic factors and survival in endocrine tumor pa-tients: comparison between gastrointestinal and pancreatic localization. Endocr Relat Can-cer 2005; 12: 1083-92.

6. Chen X, Zhao H. Gastric neuroendocrine neoplasm with liver metastases: a case report. Transl Gastroenterol Hepatol 2016; 1: 55.

7. Dakin GF, Warner RR, Pomp A, Salky B, Inabnet WB. Presentation, treatment, and outcome of type 1 gastric carcinoid tumors. J Surg Oncol 2006; 93(5): 368-72.

8. Gladdy RA, Strong VE, Coit D. Defining surgical indications for type I gastric carcinoid tumor. Ann Surg Oncol 2009; 16(11): 315460.
9. Fossmark R, Sørdal $\varnothing$, Jianu CS, et al. Treatment of gastric carcinoids type 1 with the gastrin receptor antagonist netazepide (YF476) results in regression of tumours and normalisation of serum chromogranin A. Aliment Pharmacol Ther 2012; 36: 1067-75.

10. Pritchad DM. Zollinger-Ellison syndrome still a diagnostic challenge in the $21^{\text {st }}$ century? Gas-troenterology. 2011; 140(5): 1380-83.

11. Tomassetti P, Migliori M, Caletti GC, Fusaroli P, Corinaldesi R, Gullo L. Treatment of type II gastric carcinoid tumors with somatostatin analogues. N Engl J Med 2000; 343: 551-54.

12. Namikawa T, Oki T, Kitagawa $H$, Okabayashi $T$, Kobayashi M, Hanazaki K. Neuroendocrine car-cinoma of the stomach: clinicopathological and immunohistochemical evaluation. Med Mol Mor-phol 2013; 46: 34-40.

13. Riihimäki M, Hemminki A, Sundquist K, Sundquist J, Hemminki K. The epidemiology of metas-tases in neuroendocrine tumors. Int $J$ Cancer 2016; 139(12): 2679-86.

14. Frilling A, Sotiropoulos GC, Radtke A, et al. The impact of $68 \mathrm{Ga}-$ DOTATOC positron emission tomography/computed tomography on the multimodal management of patients with neuroen-docrine tumors. Ann Surg 2010; 252: 850-6.

15. O'Toole D, Ducreux M, Bommelaer G, et al. Treatment of carcinoid syndrome: a prospective cross over evaluation of lanreotide versus octreotide in terms of efficacy, patient acceptability, and tolerance. Cancer 2000; 88: 770-6.

16. Kulke $\mathrm{MH}$, Hörsch $\mathrm{D}$, Caplin ME, et al. Telotristat Ethyl, a Tryptophan hydroxylase inhibitor for the treatment of carcinoid syndrome. J Clin Oncol 2017; 35(1): 14-23.

17. Rinke A, Wittenberg M, Schade-Brittinger $C$, et al. Placebocontrolled, double-blind, prospec-tive, randomized study on the effect of octreotide LAR in the control of tumor growth in patients with metastatic neuroendocrine midgut tumors (PROMID): results of long-term survival. Neuro-endocrinology 2017; 104: 26-32.

18. Caplin ME, Pavel M, Ćwikła JB, et al. Lanreotide in metastatic enteropancreatic neuroendo-crine tumors. N Engl J Med 2014; 371: 224-33. This phase III study provides high-level evidence that lanreotide delays progression in patients with GEP-NETs

19. Kwekkeboom DJ, Krenning EP. Peptide receptor radionuclide therapy in the treatment of neuroendocrine tumors. Hematol Oncol Clin North Am 2016; 30: 179-91.

20. Yao JC, Fazio N, Singh S, et al. Everolimus for the treatment of advanced, non-functional neu-roendocrine tumours of the lung or gastrointestinal tract (RADIANT-4): a randomised, placebocontrolled, phase 3 study. Lancet 2016; 387: 968-77.

21. Janson ET, Oberg K. Long-term management of the carcinoid syndrome. Treatment with oc-treotide alone and in combination with alpha-interferon. Acta Oncol 1993; 32: 225-9.

22. Okita NT, Kato K, Takahari D, et al. Neuroendocrine tumors of the stomach: chemotherapy with cisplatin plus irinotecan is effective for gastric poorly-differentiated neuroendocrine carci-noma. Gastric Cancer 2011; 14: 161-5.

23. Fairweather $M$, Swanson $R$, Wang $J$, et al. Management of neuroendocrine tumor liver me-tastases: long-term outcomes and prognostic factors from a large prospective database. Ann Surg Oncol 2017; 24: 2319.

24. Gupta S, Johnson MM, Murthy R, et al. Hepatic arterial embolization and chemoembolization for the treatment of patients with metastatic neuroendocrine tumors variables affecting response rates and survival. Cancer 2005; 104: 1590-1602. 